\title{
Major incidents: training for on site medical personnel
}

\author{
Colin A Graham, Stephen T Hearns
}

\begin{abstract}
Objective-To assess the present levels of training for the medical incident officer (MIO) and the mobile medical team leader (MMTL) throughout the UK. Method-Postal questionnaire to consultants in charge of accident and emergency (A\&E) departments seeing more than 30000 patients a year. Information regarding MIO staffing and training and MMTL training and provision requested. Results-A\&E provides the majority of both MIOs and MMTLs in the event of a major incident. Virtually all MIOs are consultants or general practitioners. However, $63 \%$ of MMTLs are from hospital training grade staff. One third of hospitals required their designated MIO to have undertaken a Major Incident Medical Management and Support course and a quarter had no training requirement at all. Two thirds of MMTLs were expected to have completed an Advanced Trauma Life Support course, but in $21 \%$ there was no minimum training requirement. Training exercises are infrequent, and hence the exposure of any one individual to exercises will be minimal.

Conclusion-There has been some improvement in major incident training and planning since 1992, but much remains to be done to improve the national situation to an acceptable standard.

$(\Im$ Accid Emerg Med 1999;16:336-338)
\end{abstract}

Keywords: major incidents; training; mobile medical teams; disaster planning

It is now seven years since Cooke showed the poor state of preparedness of medical personnel for key roles at the scene of a major incident. ${ }^{1}$ More recent studies have shown the inadequacy of our hospital major incident plans, ${ }^{2}$ preparation and planning for the teams (surgical and non-surgical), ${ }^{3}$ and safety of our mobile medical teams ${ }^{4}$; all this despite guidance from the NHS Management Executive. ${ }^{5}$ This is also despite increasing awareness and availability of major incident training after the introduction of the Major Incident Medical Management and Support (MIMMS) course. ${ }^{6}$ This study was undertaken to assess the present levels of training for the medical incident officer (MIO) and the mobile medical team leader (MMTL) throughout the UK.

Mr Colin Graham, Specialist Registrar, Accident and Emergency Department, Crosshouse Hospital, Kilmarnock KA2 0BE.
Methods

A questionnaire was sent in April 1997 to the named consultant in charge of each accident
Table 1 Specialties providing MIO and MMTL

\begin{tabular}{lll}
\hline Specialty & MIO (\%) & MMTL (\%) \\
\hline A\&E & $46(72 / 158)$ & $53(83 / 156)$ \\
GP & $24(38 / 158)$ & $3(5 / 156)$ \\
Anaesthetics & $13(20 / 158)$ & $21(32 / 156)$ \\
General medicine & $11(18 / 158)$ & $4(6 / 156)$ \\
Orthopaedics & $11(17 / 158)$ & $17(26 / 156)$ \\
Gynaecology & $5(8 / 158)$ & - \\
General surgery & $4(6 / 158)$ & $26(40 / 156)$ \\
Other & $17(27 / 158)$ & $7(11 / 156)$ \\
\hline
\end{tabular}

and emergency (A\&E) department in the UK seeing more than 30000 patients a year. Appendix 1 details the questionnaire. The departments were identified from the British Association for Accident \& Emergency Medicine's 1997 directory. Non-responders were sent a single reminder letter and a further copy of the questionnaire eight weeks after the initial mailing. Altogether 232 questionnaires were sent out and 192 returned in total, giving a response rate of $83 \%$. Thirty of the hospitals were not designated to provide a mobile medical team in the event of a major incident and were not analysed further. There were therefore 162 questionnaires for analysis, but as not all questions were answered by all respondents, total respondents for each question are included in the results.

Information about the MIOs and the MMTLs designated by each of the hospital major incident plans was requested; this included their grade, specialty, and the minimum training requirements for them to undertake these roles. As the majority of hospitals have a number of people designated for these positions those completing the questionnaire were not restricted to one answer per question.

"Minimum training requirements" were not expressly specified on the questionnaire, but are taken to be those specified in the major incident plan for that $A \& E$ department. Similarly, the definition of "regular experience of pre-hospital care" was left to the discretion of the respondent, but was taken to mean involvement in a local immediate care scheme or $\mathrm{A} \& \mathrm{E}$ flying squad.

\section{Results}

SPECIALTY AND GRADE OF THE MIO AND MMTL Half of the hospitals surveyed have designated MIOs and MMTLs from the A\&E department, as seen in table 1 . Surgeons, both general and orthopaedic, are designated the task of MMTL in $42 \%(66 / 156)$ of the plans. Almost a quarter of major incident plans involved general practitioners (GPs) as designated MIOs, though GPs are rarely allocated the role of MMTL (4\%, 6/157). 
Table 2 Minimum training requirements for $M I O$ and $M M T L$

\begin{tabular}{lll}
\hline Training & MIO (\%) & MMTL (\%) \\
\hline ATLS & $39(59 / 151)$ & $63(97 / 155)$ \\
MIMMS & $36(55 / 151)$ & $17(26 / 155)$ \\
PHTLS & $10(15 / 151)$ & $7(11 / 155)$ \\
DipIMC & $5(7 / 151)$ & $1(2 / 155)$ \\
Local teaching & $34(52 / 151)$ & $31(48 / 155)$ \\
None & $25(38 / 151)$ & $21(33 / 155)$ \\
\hline
\end{tabular}

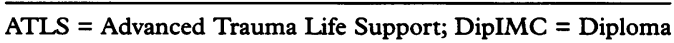
in Immediate Medical Care; MIMMS = Major Incident Medical Management and Support; PHTLS = Pre-hospital Trauma Life Support.

The vast majority $(98 \%, 156 / 159)$ of designated MIOs are of GP or consultant grade, with a small number coming from the training grades. However $63 \%(99 / 157)$ of plans have MMTLs from hospital training grade staff.

TRAINING REQUIREMENTS AND EXPERIENCE OF THE MIO AND MMTL

The hospitals surveyed varied widely in the minimum training required of those undertaking the role of MIO as can be seen in table 2. Only one third of hospitals required their designated MIO to have undertaken a MIMMS course and a quarter had no minimum training requirements at all.

Standards of training expected of potential MMTLs were higher, with almost two thirds expected to have completed an Advanced Trauma Life Support provider course. Again, however, $21 \%(33 / 155)$ of plans have no minimum requirements for those expected to undertake such a role.

The study revealed that of the hospitals surveyed, $55 \%(86 / 155)$ of designated MIOs and $68 \%(107 / 157)$ of MMTLs did not have regular experience of pre-hospital care. The majority of hospitals $(84 \%)(135 / 161)$ undertake training exercises involving the other emergency services; however, in $35 \%(45 / 129)$ of these the exercises occur every two years and in $16 \%(21 / 129)$ exercises occur less frequently than this.

The survey also showed that two thirds $(64 \%)(101 / 158)$ of the major incident plans of those responding did not involve local GP immediate care schemes. This is not surprising as only one third of the country are currently covered by formal immediate care schemes.

\section{Discussion}

Medical personnel called upon to work at the scene of a major incident are required to operate in an unfamiliar, hostile, and inherently dangerous environment. They must work in cooperation with and rely upon the other emergency services, and make use of unfamiliar communication media. The work they must do and the place they are doing it are far removed from their everyday clinical practice. They must make demanding clinical and management decisions and carry out practical procedures in this environment while contending with limited assistance, resources, and equip- ment. Therefore it is essential for their personal safety and effectiveness that they are adequately trained and practice regularly for this role.

It is clear that, while significant improvements have been made since 1992, much remains to be done. It is encouraging that the majority of those designated the role of MIO are senior clinicians from acute specialties. However it is of concern that a quarter of hospitals have no minimum training requirements for MIOs and that only $36 \%$ demand completion of a MIMMS course. It is also discouraging that MIOs and MMTLs from many hospitals are so infrequently involved in major incident exercises and pre-hospital care. Given that exercises are infrequent and MIOs are likely to be on a one in five rota at worst, any particular MIO will be called to participate in an exercise once in every 10 years (assuming exercises every two years). This cannot be adequate training for the demanding and unusual role of $\mathrm{MIO}$.

It is surprising that only a minority of plans utilise the skills of general practice based immediate care doctors, who are used to working in the pre-hospital environment, ${ }^{7}$ and who are aware of the resources of local hospitals where casualties can be dispatched. Furthermore, if the MIO or MMTL is drawn from the hospital closest to the major incident, this will deplete the base hospital of vital staff at a crucial time in its response. This lends weight to the argument to use GP based immediate care doctors or $A \& E$ consultants from an "out of area" location as MIOs in the event of a major incident.

It is time for major incident planners to reconsider the level of training and expertise required of their potential MIOs and MMTLs and revise their major incident plans and requirements for training accordingly.

We would like to thank all the consultants in A\&E medicine who completed and returned the questionnaires. We would like to thank the Clinical Audit Department at Glasgow Royal Infirmary for extensive assistance with data analysis. We also thank Dr Chris Rowlands, Mr Ian Swann, and Miss Patricia Clark for their assistance.

Conflict of interest: none.

Funding: the study was supported by a grant from the Greater Glasgow Health Board Endowment Fund.

1 Cooke MW. Arrangements for on scene medical care at major incidents. $B M \Im 1992 ; 305: 748$.

2 major incidents. BMF 1992;305:748. Carley S, Mackway-Jones $\mathrm{K}$. Are British hospitals ready for
the next major incident? Analysis of hospital major incident plans. BMF 1996;313:1242-3.

3 Carley SD, Mackway-Jones K. The preparation for the prehospital surgical and non-surgical response to major incidents in the United Kingdom. Pre-hospital Immediate Care 1997;1:68-70.

4 McGregor P, Driscoll P, Sammy I, et al. Are UK mobile medical teams safe? Pre-hospital Immediate Care 1997;1: 183-8.

5 NHS Management Executive. Emergency planning in the NHS: health services arrangements for dealing with major incidents. London: NHSME, 1990 HC(90)25.

6 Advanced Life Support Group. Major incident medical management and support. London: BMJ Publishing Group, 1996.

7 Fisher JM. The British Association for Immediate Care (BASICS). Its experience in major disasters, with special reference to the role of the medical incident officer. Injury 1990;21:45-8. 


\section{Appendix 1 Questionnaire used for major incident study}

Part 1-Medical incident officer (MIO)

$1 . \quad$ Is your hospital designated to provide a mobile medical team?

Yes/no

2. In your major incident plan, what specialty (ies) is your MIO from?

A\&E/GP (immediate care)/orthopaedic surgeon/general surgeon/physician/other (specify)

3. What grade is the MIO?

GP/consultant/SpR/ other (specify)

4. What are the minimum training requirements for your MIO?

MIMMS/ATLS / PHTLS/DipIMC /DMCC/none/local teaching (specify)

5. Does the MIO have regular experience of pre-hospital care? (for example, immediate care scheme or flying squad) Yes/no

Part 2-Mobile medical team

6. In your major incident plan, what specialty provides the mobile medical team leader (MMTL)?

A\&E/GP (immediate care)/orthopaedic surgeon/general surgeon/physician/other (specify)

7. What grade is the MMTL ?

GP/consultant/SpR/other (specify)

8. What are the minimum training requirements for the MMTL

MIMMS/ATLS/PHTLS/DipIMC/DMCC/none/local teaching (specify)

9. Does training involve exercises with the other emergency services?

Yes/no

IF YES: How often?

10. Does the MMTL have regular experience of pre-hospital care? (for example, immediate care scheme or flying squad) Yes/no

11. Does your plan involve local immediate care doctors (GPs)? Yes/no

Thank you for completing this questionnaire

ATLS = Advanced Trauma Life Support; DipIMC = Diploma in Immediate Medical Care; DMCC = Diploma in the Medical Care of Catastrophes; MIMMS = Major Incident Medical Management and Support; PHTLS = Pre-hospital Trauma Life Support; SpR $=$ specialist registrar. 